\title{
Genomic profiling of the genes on chromosome 3p in sporadic clear cell renal cell carcinoma
}

\author{
YOSHIKAZU TOGO $^{1 *}$, YOSHIE YOSHIKAWA ${ }^{2 *}$, TORU SUZUKI ${ }^{1}$, YOSHIRO NAKANO ${ }^{2}$, \\ AKIHIRO KANEMATSU ${ }^{1}$, MASATAKA ZOZUMI ${ }^{3}$, MICHIO NOJIMA ${ }^{1}$, SEIICHI HIROTA ${ }^{3}$, \\ SHINGO YAMAMOTO $^{1}$ and TOMOKO HASHIMOTO-TAMAOKI ${ }^{2}$ \\ Departments of ${ }^{1}$ Urology and ${ }^{2}$ Genetics, ${ }^{3}$ Division of Surgical Pathology, Department of Pathology, \\ Hyogo College of Medicine, Hyogo 663-8501, Japan
}

Received December 2, 2015; Accepted January 28, 2016

DOI: 10.3892/ijo.2016.3395

\begin{abstract}
Somatic mutations of the BRCA1 associated protein-1 (BAPl) gene, which maps to 3p21, have been found in several tumors including malignant mesothelioma, uveal melanoma, and renal cell carcinoma (RCC). The role of BAP1 inactivation in tumor development remains unclear. It has been reported that $V h l$ knock-out mice did not develop RCC, but $V h l$ knock-out mice with single allele loss of Bapl in nephron progenitor cells developed RCC, indicating that Bap1 inactivation may be essential in murine renal tumorigenesis. To clarify the role of BAP1 in human RCC development, we performed mutation analyses, including copy number detection of $B A P I$ and assessment of allelic imbalance using microsatellite polymorphisms on 3p, in 45 RCC samples derived from 45 patients without $V H L$ or $B A P 1$ germline mutation. Additionally, we analyzed the sequences of the $V H L, P B R M 1$, and SETD2 genes, and examined promoter methylation of $V H L$. Using immunostaining, we also checked for expression of BAP1 protein, which is normally located in the nuclei. None of the RCCs had biallelic deletion of $B A P 1$, but five (11.1\%) showed a biallelic mutation (four with a sequence-level mutation with monoallelic loss and one with a biallelic sequence-level mutation); these cells were negative for nuclear BAP1 staining. These patients had worse recurrence-free survival than the patients without a biallelic mutation $(\mathrm{p}=0.046)$. However, there were no significant differences in worse outcome by multivariate analysis combined with age, $\mathrm{T}$ stage, histological subtype, infiltration and vascular invasion. In 35 RCCs (77.8\%), monoallelic loss of $B A P I$ was accompanied by $V H L$ biallelic mutation or $V H L$ promoter hypermethylation. In five RCCs
\end{abstract}

Correspondence to: Dr Tomoko Hashimoto-Tamaoki, Department of Genetics, Hyogo College of Medicine, Mukogawa-cho, 1-1 Nishinomiya, Hyogo 663-8501, Japan

E-mail: tomokots@hyo-med.ac.jp

*Contributed equally

Key words: renal cell carcinoma, von Hippel-Lindau tumor suppressor, BRCA1 associated protein-1, loss of heterozygosity, monoallelic loss
(11.1\%), we detected 3p loss-of-heterozygosity, but the copy number of $B A P 1$ was normal. Surprisingly, nuclear staining of BAP1 was negative in 10 out of 31 tumors $(32.3 \%)$ with hemizygous normal $B A P 1$, suggesting that haploinsufficiency may relate to RCC development.

\section{Introduction}

Loss of chromosome $3 p$ in many tumors is well established and it has been supposed for a long time that this chromosomal region might carry several tumor suppressor genes $(1,2)$. In particular, sporadic clear-cell renal cell carcinoma (ccRCC) is characterized by a high frequency of allelic deletion or loss of heterozygosity (LOH) on chromosome $3 p(>90 \%)$, causing biallelic inactivation of von Hippel-Lindau tumor suppressor $(V H L)$ gene (3-5). It was reported that deletion of fragile histidine triad (FHIT) gene and flanking loci might occur as an initiating event followed by deletions at $3 \mathrm{p} 12.2$, $3 \mathrm{p} 21.31-3 \mathrm{p} 21.32$, and $3 \mathrm{p} 24.2-3 \mathrm{p} 26.1$ in the early stages, then continuous large deletions of 3p21.3-3p26.1 and 3p14.1-3p26.1 (6). Next-generation sequencing studies have identified frequent mutations in genes involved in chromatin modification such as polybromo 1 (PBRMI), SET domain containing 2 (SETD2), and BRCA1 associated protein-1 (BAP1) (7-10), all of which are located on $3 \mathrm{p} 21$. Moreover, $B A P 1$ mutations were mutually exclusive with $P B R M 1$ mutations $(9,11)$.

Germline BAPl mutations are associated with an increased risk of malignant mesothelioma (MM), atypical melanocytic tumors, and other neoplasms (12-15). They also predispose to RCC $(16,17)$. Moreover, somatic BAPI mutation was reported in $\sim 10 \%$ of ccRCC patients of any ethnic background $(9-11,18)$. Recently, Wang et al reported that mice deficient for Vhl together with one allele of Bap1 in nephron progenitor cells developed ccRCC, but mice deficient in Vhl did not (19). Wang et al suggested that BAPI is a more potent tumor suppressor gene than VHL, which they assessed as a weak tumor suppressor gene in the kidney. The question remains as to why VHL mutations occur at a much higher frequency than BAPI mutations in both familial and sporadic ccRCC (20).

The BAP1 protein is a nuclear protein that has been considered to function as a deubiquitinase of histone $\mathrm{H} 2 \mathrm{~A}$ (21). Two types of BAP1 inactivation have been reported: sequence- 
level mutation of BAP1 combined with monoallelic loss of $3 p$, and biallelic deletion comprising broad deletions of $3 \mathrm{p} 21$ and narrow deletions of several exons or the entire $B A P I$ gene. In metastasized uveal melanoma, the former mutation type occurs frequently $(22,23)$. In MM, we reported a high frequency of biallelic loss of $B A P l(24,25)$, in addition to sequence-level mutations in the $B A P 1$ gene (26). However, the observed frequency of BAP1 inactivation in MM has been variable among studies: low frequencies of mutations found by sequencing alone $(12,26,27)$, and high frequencies of loss of nuclear staining, or genomic mutations identified by sequencing and copy number analysis $(24,28,29)$. Nasu et al suggested immunostaining as the most accessible and reliable technique to detect BAP1 inactivation in MM biopsies (29). It was also reported that BAP1 expression in the nucleus was reduced in $44 \%$ of esophageal squamous carcinoma, although the mutation rate of the gene was $2 \%$ (30). It is increasingly clear that BAP1 protein nuclear translocation may play a key role in tumorigenesis $(31,32)$.

To clarify the status of inactivation of the $B A P l$ gene in ccRCC development, we performed genomic analysis of the $B A P 1$ gene, assessed for $3 \mathrm{p}$ rearrangement, and conducted immunostaining for the BAP1 protein in RCC patients.

\section{Materials and methods}

Tumor specimens. We obtained 45 RCC samples from 45 patients who had radical nephrectomy at the Hospital of Hyogo College of Medicine. The patient summary is presented in Table I. Fresh frozen tumor tissues dissected from the surgical specimen and unaffected kidney tissue adjacent to the tumor regions for most patients (ID: RCC01-56), or peripheral blood for 13 patients (ID: RCC57-69), as a matched control were analyzed. Pathological examination showed that 42 tumors were diagnosed as ccRCC and three as chromophobe RCC (chRCC). Our study was approved by the Ethics Committee of Hyogo College of Medicine (permission no.: RINHI277), and performed in accordance with the Declaration of Helsinki (1995) of the World Medical Association (as revised in Fortalez, 2013). All patients provided written informed consent.

Microsatellite (MS) genotyping of chromosome $3 p$. We analyzed 13 polymorphic MS markers, identified from the 'Heterozygosity of MS Markers in the Japanese Population' database (https://dbarchive.biosciencedbc.jp/jp/heterozy gosity-jp/desc.html), using a modified version of the method described by Dietrich et al (33). We examined the following markers: D3S1263 (3p25.3); D3S1266 (3p24.1); D3S1611 (3p22.2); D3S3687 (3p22.1); D3S3678 (3p22.1); D3S2420 (3p21.31); D3S1568 (3p21.31); D3S1573 (3p21.31); D3S3561 (3p21.1); D3S3648 (3p21.1); D3S1289 (3p14.3); D3S1300 (3p14.2); and D3S1285 (3p14.1). When the locus was heterozygous in the matched control sample, we calculated the ratio of the peak area of allele 1 compared with that of allele 2 , and determined the allelic imbalance between the allele ratio of the tumor and that of the matched control sample. We judged $\mathrm{LOH}$ and deduced the rate of contaminating non-tumor cells included in RCC tumors based on the value of allelic imbalance. The rates of tumor content were lower than the rates estimated by pathological examination.
Multiplex ligation probe amplification (MLPA) analysis. We carried out MLPA analysis of genomic DNA for each of the 17 exons of the BAPl gene using a SALSA MLPA BAP1 kit according to the manufacturer's instructions (MRC Holland, Amsterdam, The Netherlands). This kit detects copy number changes in each exon of $B A P l$ plus an additional ten genes on chromosome 3: MLH1, RBM5, RASSF1, ZMYND10, HESX1, FHIT, MITF, ROBOl, PROS1 and CPOX. The peak area obtained using gene-specific probes in each sample was first normalized to the average peak areas obtained using control probes specific for other chromosomal locations. A final ratio was then calculated by dividing by the value for genomic DNA isolated from the matched control. The sample was scored to have loss or gain of one copy number (monoallelic loss or amplification) by comparing the MLPA ratio and the rate of tumor content in the RCC sample. For example, if the rate of tumor content calculated by MS analysis and the MLPA ratio were 0.5 and 0.75 , respectively, this would indicate monoallelic loss of BAPl in an RCC sample that consisted of $\leq 50 \%$ non-tumor cells.

Target sequencing. We isolated DNA using an AllPrep DNA/ RNA mini kit (Qiagen, Germantown, MD, USA) according to the manufacturer's instructions, and sequenced the coding regions of $V H L, B A P 1, S E T D 2$ and $P B R M 1$. For $V H L$, direct sequencing was conducted using a BigDye Terminator v3.1 kit on an Applied Biosystems 3130xl Genetic Analyzer (Foster City, CA, USA); primer sequences are presented in Table II. By consulting the contamination rate of non-tumor cells in each tumor sample, a barely imperceptible variant in the tumor sample could be detected by sequencing on both strands. For the other genes, next-generation sequencing was performed on an Illumina MiSeq (San Diego, CA, USA) using paired-end 250-bp runs. Libraries were prepared from $250 \mathrm{ng}$ of genomic DNA from 45 independent RCC tumors using a Haloplex Custom kit (Agilent Technologies, Santa Clara, CA, USA) according to the manufacturer's instructions. Illumina paired-end reads were each aligned to the human NCBI Build 37 reference sequence using bwa software (biobwa.sourceforge.net/, version 0.6.0). The aligned sequence files were sorted and merged using SAMtools (samtools. sourceforge.net/, version 0.1.18). GATK (http://www.broad institute.org/gatk/) was used for realignment, base quality score recalibration, single nucleotide variant or indel (small insertions and deletions) variant calling, and variant quality recalibration. SnpEff was used to categorize the effects of variants by impact (34). Functional annotation of genetic variants provided by ANNOVAR (www.openbioinformatics.org/ annovar/, version 2013, Feb 11) and dbNSFP (35) were used to identify protein-damaging mutations. Somatic mutations or germline variants were judged by comparison between Sanger sequence data from the ccRCC tumor and its matched control.

Promoter methylation of VHL. Bisulfite sequencing was performed for the patients who did not have a mutation in the VHL gene: ID 5, 6, 7, 21, 23, 36, 44, 55 and 67. After bisulfite modification of $100 \mathrm{ng}$ tumor or normal DNA using an EpiTect Fast DNA Bisulfite kit (Qiagen), the regions containing both methylated and unmethylated alleles across $26 \mathrm{CpG}$ dinucleotides in the $V H L$ promoter were amplified using a touchdown 


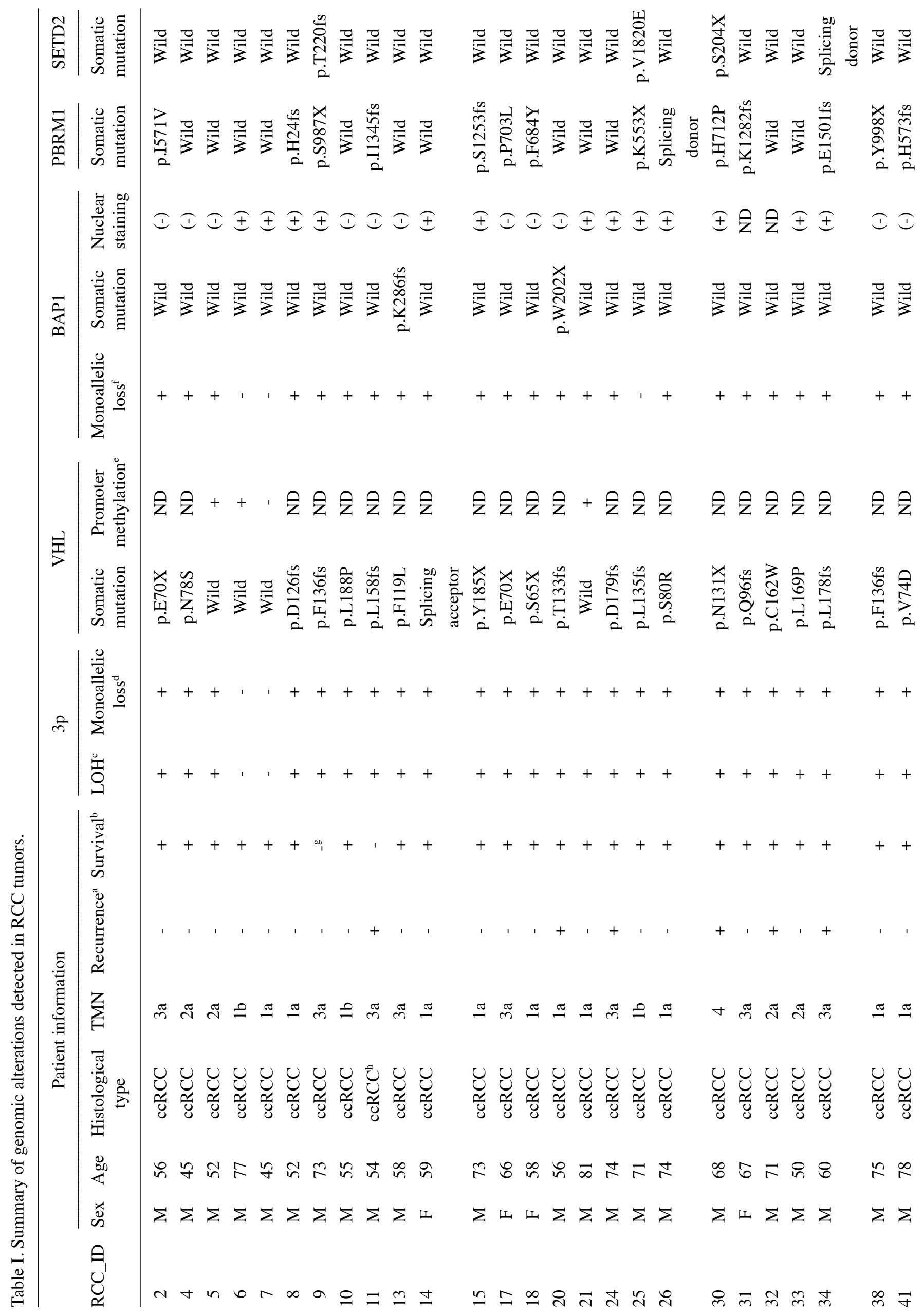




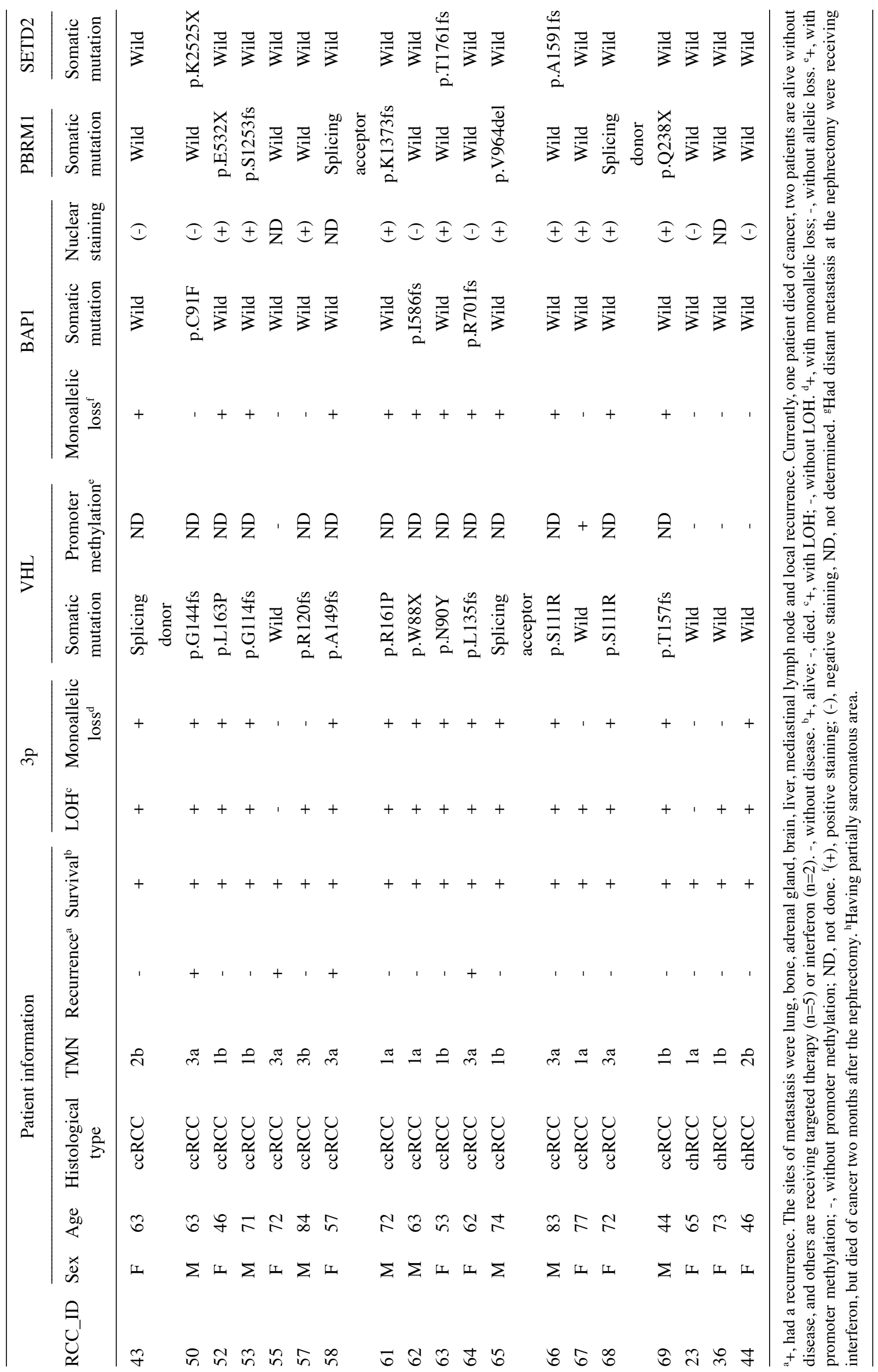




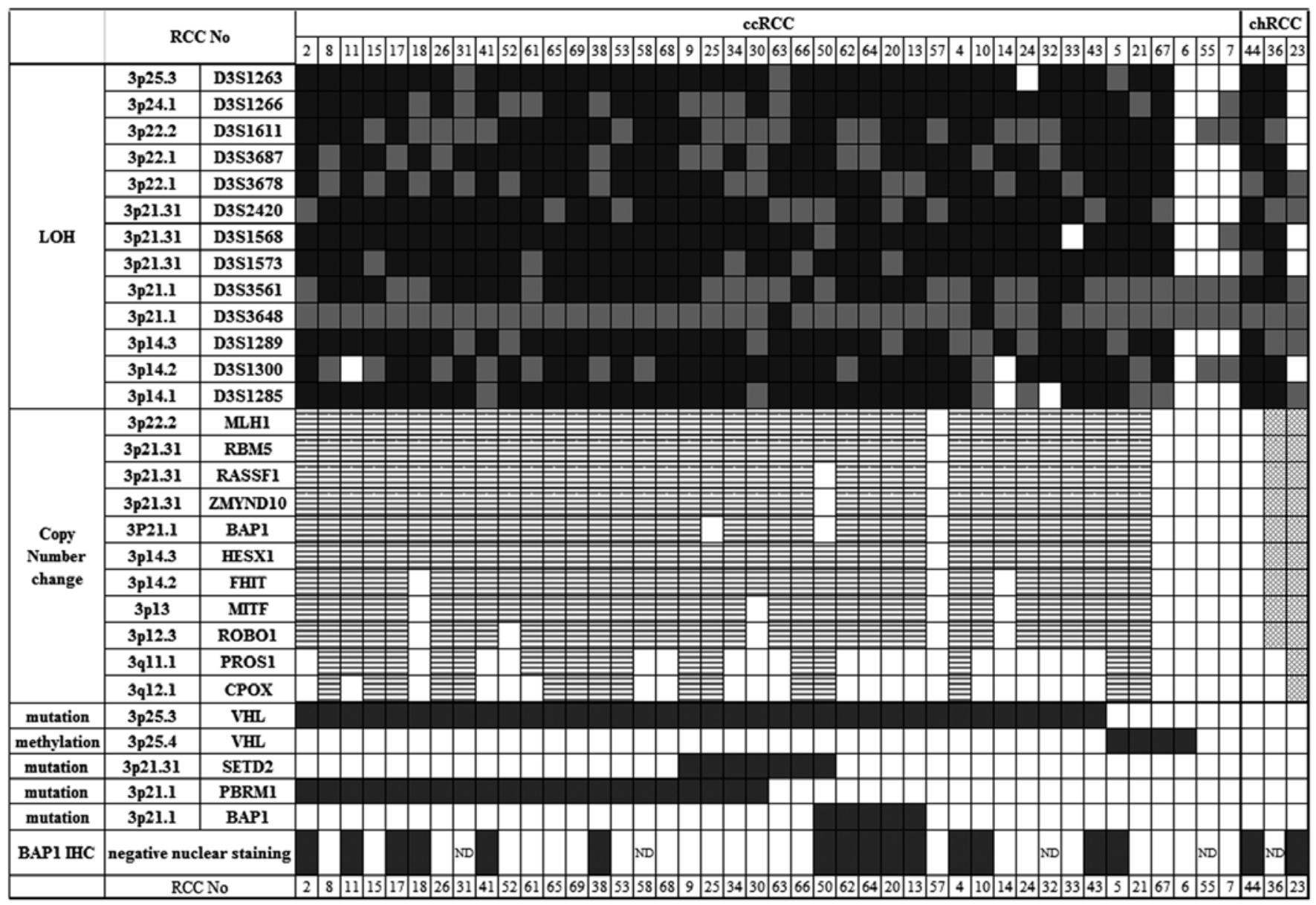

Figure 1. Summary of genome alterations and BAP1 protein expression in renal cell carcinomas. A solid fill indicates that the tumor had the corresponding alteration with respect to the matched control. Loss of heterozygosity $(\mathrm{LOH})$, black fill indicates $\mathrm{LOH}$, gray fill indicates a homozygous genotype in germline DNA, in which case LOH could not be judged in the tumor. Copy number change, horizontal lines indicate loss of one copy and hatched lines indicate gain of one copy. BAP1 immunohistochemistry, a solid fill indicates negative staining for nuclear BAP1 protein. 'ND' in the nuclear staining column indicates that a call could not be made because the tumors had focal negative or weak positive expression.

PCR program with an annealing temperature of $50^{\circ} \mathrm{C}$ : 1st PCR primers: 5'-TTAYGGAGGTYGATTYGGGAG-3' and 5'-ACRATTACAAAAAATAACCTA-3' (amplicon: 335 bp); nested PCR primers: 5'-YGGGTGGTTTGGATYG-3' and 5'-AATTCACCRAACRCAACA-3' (amplicon: 226 bp), as previously reported (36). The cytosine positions in $\mathrm{CpGs}$ were inspected for thymine or cytosine signals on the chromatograms. Promoter methylation was not detected with normal samples; tumor samples with $\geq 30 \%$ methylated CpGs were judged as methylated.

Nuclear staining. RCC tissues embedded in paraffin were cut into 3- $\mu \mathrm{m}$-thick sections and heated in antigen retrieval solution (Bond Epitope Retrieval Solution 2, pH 9.0; Leica Biosystems, Nussloch, Germany) at $98^{\circ} \mathrm{C}$ for $10 \mathrm{~min}$. Then, the sections were incubated with mouse monoclonal antibody against human BAP1 (C-4; sc-28383; Santa Cruz Biotechnology, Santa Cruz, CA, USA; 1:150 dilution). Bond Polymer Refine Detection (Leica Biosystems) was used for visualization with 3, 3'-diaminobenzidine tetrahydrochloride. Substantial tumors had heterogeneous nuclear staining for BAP1, with some cells staining positively and some cells staining negatively. Negativity was evaluated when the majority of tumor cell nuclei were stained negatively while there was obvious staining in the nuclei of normal renal tubule cells on the same slide. A pathologist reviewed all slides and categorized tumors as positive, negative, or weak-positive. After samples with weak-positive expression were excluded, 40 samples were available for analysis (Table I).

Statistical analysis. Survival curves were calculated by the Kaplan-Meier method and compared by log-rank test. Two patients died of cancer, therefore the analysis for overall survival was not done. All p-values were two-tailed, and differences were considered significant at $\mathrm{p}<0.05$.

\section{Results}

Ninety-five percent of ccRCC tumors had either biallelic mutation or promoter methylation of VHL, while chRCCs did not. A high frequency, 36/45 (80\%), of VHL mutation was detected in RCC tumors by Sanger sequencing. The mutation type was: nonsense in six, frameshift in 15 , splice site in three, and missense in 12 (Table I). The RCC tumors with $V H L$ mutation showed LOH on 3p (Fig. 1). No patients had germline mutation of this gene (data not shown). Promoter methylation 
Table II. Sequences of the primers used for the direct sequencing of the VHL gene.

\begin{tabular}{|c|c|c|c|}
\hline Exon & Uses for PCR or sequencing & Forward or reverse & Sequence \\
\hline \multirow[t]{2}{*}{1} & PCR and sequencing & Forward & TGGAAATACAGTAACGAGTTGGC \\
\hline & PCR and sequencing & Reverse & GCTTCAGACCGTGCTATCGT \\
\hline \multirow[t]{3}{*}{2} & PCR & Forward & GGAGAAAATAGGTGCCCTGAC \\
\hline & PCR and sequencing & Reverse & GGCAAAAATTGAGAACTGGGCT \\
\hline & Sequencing & Forward & CCAAAGTGCTGGGATTACAGG \\
\hline \multirow[t]{3}{*}{3} & PCR & Forward & GGGGGCCATCAGCATAACAC \\
\hline & PCR and sequencing & Reverse & TACTTCTCTAATGGGCAGGCA \\
\hline & Sequencing & Forward & GTAGTTGTTGGCAAAGCCTC \\
\hline
\end{tabular}

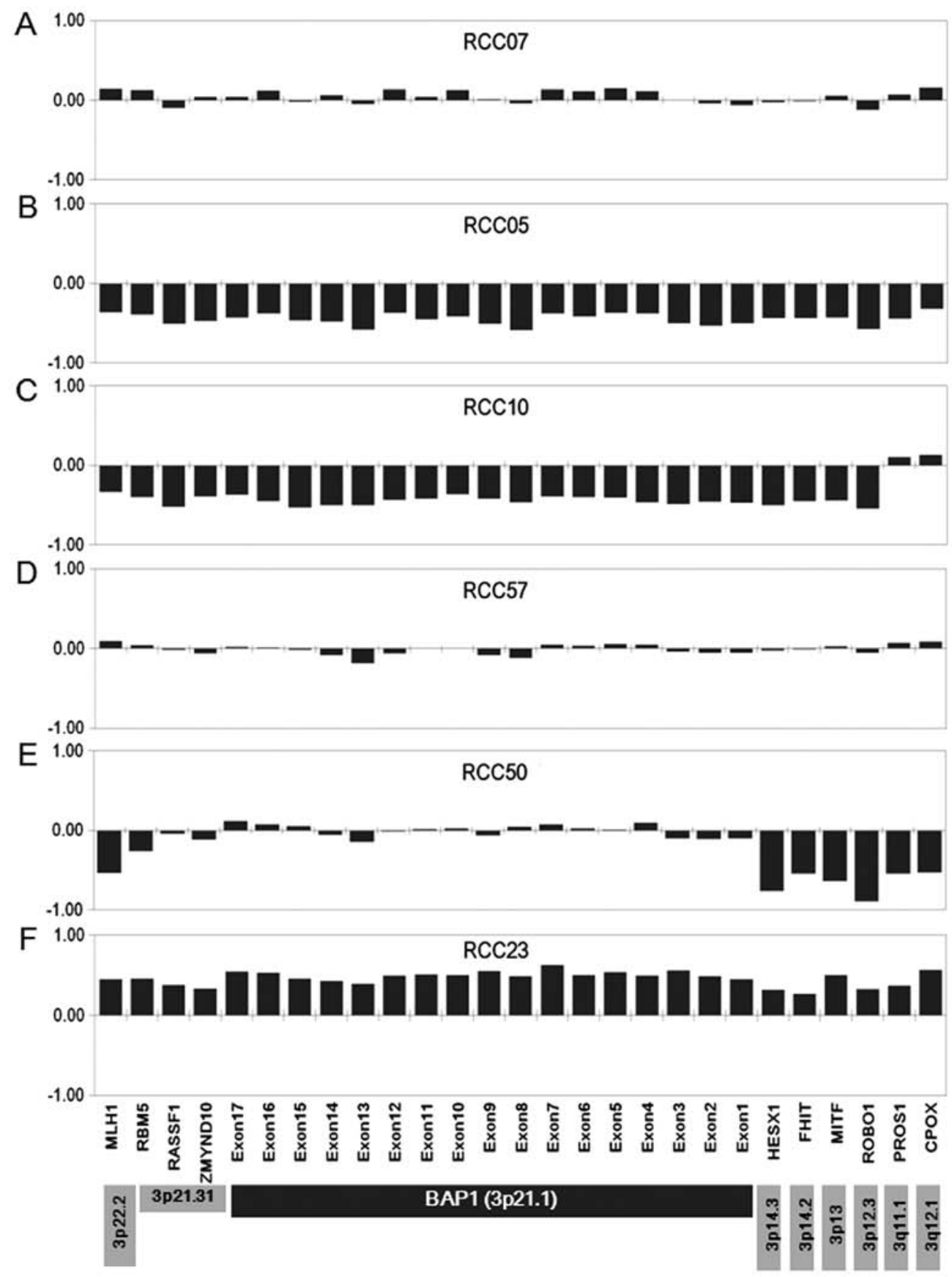

Figure 2. MLPA data for all 17 exons of the BAP1 gene on 3p21.1. The data of BAP1, as well as for 10 genes on chromosome 3p and chromosome 3q, are displayed on the x-axis. The $\log _{2}$ ratio of MLPA data for each probe is indicated on the y-axis. From top to bottom, (A) RCC07 without 3p LOH; (B and C) RCC05 and 10 , respectively, with LOH and monoallelic loss of 3p; (D) RCC57 with LOH but not monoallelic loss of 3p; (E) RCC50 with 3p LOH but not monoallelic loss of the $B A P 1$ locus; (F) RCC23 showing amplification of 3p. 


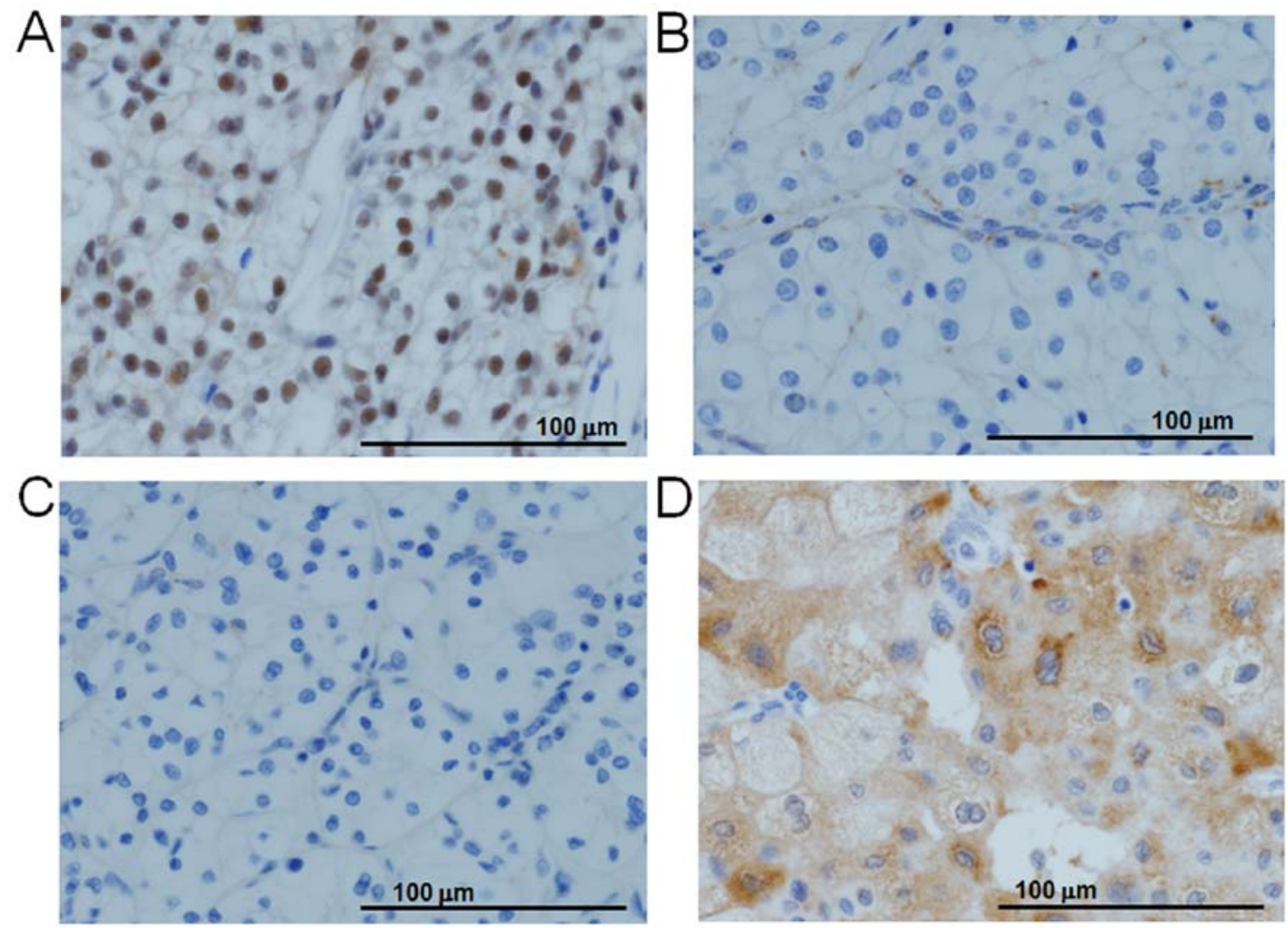

Figure 3. BAP1 nuclear staining by immunohistochemistry. (A) RCC09 showing positive nuclear staining; (B) RCC20 with biallelic mutation showing negative staining; (C) RCC02 with hemizygous normal BAPl showing negative staining; (D) chRCC23 showing negative nuclear staining but strong cytosolic staining. Scale bars represent $100 \mu \mathrm{m}$.

of $V H L$ was detected in four tumors: three (RCC05, 21 and 67) with LOH and RCC06 without LOH. Especially prominent hypermethylation was detected in RCC06 and RCC67 (data not shown). By pathological examination, three (RCC23, 36 , and 44) out of five tumors without $V H L$ mutation were noted to be chRCC. All tumors with either somatic mutation or promoter methylation in $V H L$ were from patients with ccRCC.

Seventy-eight percent of RCC tumors had monoallelic loss of $B A P 1$, but none showed biallelic loss of BAPl. All selected MS markers had a high frequency of heterozygosity in the Japanese population except for D3S3648; because this marker is close to the $B A P l$ locus, the homozygosity rate was high [42 of our 45 subjects (93.3\%)], and LOH was mostly not determined for the D3S3648 region. Instead, we deduced LOH of the $B A P 1$ region by combining the $\mathrm{LOH}$ data of flanking loci with copy number data determined by comparison of RCC tumor and matched control samples.

We did not find biallelic loss of BAPl in RCC samples. LOH in $3 p$ was detected at a frequency of $41 / 45(91 \%$; Table I and Fig. 1), and using MLPA analysis we identified monoallelic loss of all exons of BAPl together with genes on 3p22.2-3p14.3 in 35 of 45 tumors (77.8\%; Figs. 1, 2B and 2C). Two ccRCCs (RCC14 and 18) demonstrated loss of BAPl, but not FHIT nor genes on 3p13-12. Although showing 3p LOH, three tumors that had no changes in copy number to any $B A P I$ exons and any genes analyzed on $3 \mathrm{p}$ were judged to show uniparental disomy: RCC44, 57 and 67 (Figs. 1 and 2D). Two tumors (RCC25 and 50) retained two copies of $B A P 1$ although other genes on $3 \mathrm{p}$ had lost one copy (Fig. 2E). In two chRCCs (RCC23 and 36), MLPA indicated amplification of all exons of $B A P 1$ together with other genes on 3p (Fig. 2F), although gross copy number changes among the genome regions designed as normalization probes (13 control probes specific to chromosomes other than chromosome 3 ) were also detected (data not shown).

Biallelic inactivation of BAPI was detected in five ccRCCs, but not combined mutation of both BAPI and PBRMI. Somatic mutation in $B A P I$ was detected in five ccRCCs with $\mathrm{LOH}$ (mutation frequency 11.1\%); three ccRCCs had a frameshift mutation (RCC13, 62, and 64), one had a nonsense mutation (RCC20), and one had a missense change at amino acid Cys91, which is essential for ubiquitin C-terminal hydrolase activity (RCC50) (http://www.uniprot.org/uniprot/Q92560). Thus, all mutations detected in $B A P 1$ were predicted to cause loss-offunction. The mutation frequencies of PBRM1 and SETD2 in our RCCs were 21/45 (46.7\%), and 7/45 (15.6\%), respectively (Table I and Fig. 1). Only RCC50 had a mutation in both $B A P I$ and SETD2. Somatic mutations in both $B A P 1$ and PBRMI were not detected; this combined mutation has been reported to be rare.

Approximately 1/3 of ccRCCs with hemizygous normal BAPI showed negative nuclear staining. BAP1 nuclear staining was negative in the tumors with biallelic mutation of $B A P I$ (Fig. 3B). In addition, BAP1 nuclear staining was negative in 10 ccRCCs with hemizygous normal BAPI (10/31, 32.3\%) 


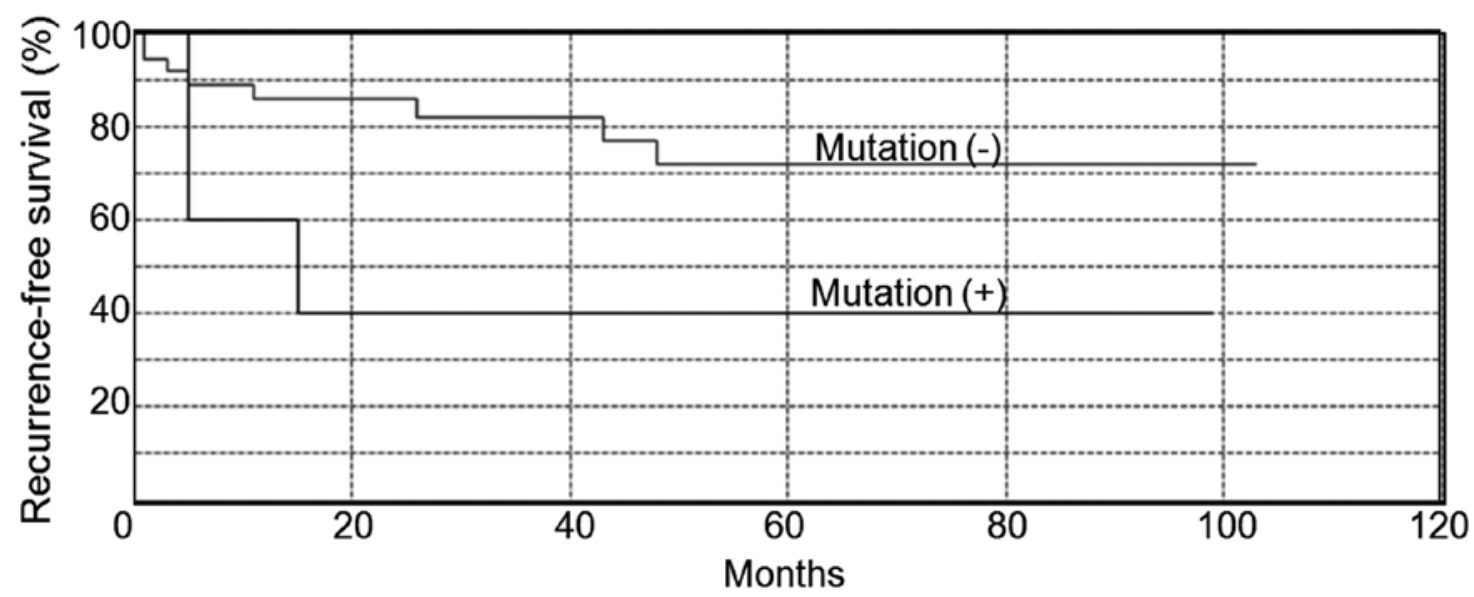

Figure 4. Impact of $B A P 1$ mutation on recurrence-free survival time. Survival curves were calculated by the Kaplan-Meier method and compared by log-rank test. All p-values were two-tailed, and differences between 'with' [mutation (+)] and 'without' [mutation (-)] BAP1 mutation were considered significant at $\mathrm{p}<0.05(\mathrm{p}=0.046)$.

(Figs. 1 and 3C). Three chRCCs had strong BAP1 staining in the cytosol (Fig. 3D), although two of them were negative and one was excluded from analysis with nuclear staining.

Biallelic inactivation of BAPl led to worse outcome. We investigated the relationship between $B A P 1$ mutation and tumor recurrence. The ccRCC tumors with biallelic loss-offunction mutation in $B A P 1$ correlated with recurrence-free survival time ( $\mathrm{p}=0.046$, Fig. 4 ), but not by multivariate analysis combined with age, $\mathrm{T}$ stage, histological subtype, infiltration and vascular invasion (data not shown). SETD2 mutations significantly correlated with recurrence-free survival time $(\mathrm{p}=0.007)$, but PBRM1 mutations did not $(\mathrm{p}=0.761)$ (data not shown). The ccRCC tumors with negative BAP1 nuclear staining did not associate with tumor recurrence $(p=0.143$, data not shown).

\section{Discussion}

We confirmed that $95 \%$ of our ccRCCs had features of the VHL-dependent pathway of tumorigenesis. VHL inactivation (mutation by base substitution or indel, or promoter methylation) perfectly paralleled $3 p \mathrm{LOH}$ in ccRCC tumors. It is known that point mutations in $V H L$, and loss of $3 \mathrm{p}$, which has been suggested to associate with the deletion of FHIT located on 3 p14.2 $(5,6)$, are early events during tumorigenesis and are thought to initiate ccRCC development. Tumors from two stage I ccRCC patients (RCC14 and 18) had monoallelic loss of BAPl together with genes on 3p22.2-3p14.3, but not FHIT. Deletion of FHIT might not be essential for a large loss of $3 \mathrm{p}$, but mutations of several tumor suppressor genes on $3 \mathrm{p}$ might trigger a large loss of $3 \mathrm{p}$ by cooperating with $V H L$ mutation.

Mutations in BAP1, PBRM1 and SETD2 were detected in ccRCCs with $V H L$ mutation. The rate of hemizygous truncation mutation of $B A P 1$ in ccRCC was $4 / 42$, resulting in negative nuclear expression of the encoded protein. RCC50, which showed uniparental disomy on chr3p21.31-3p21.1, had a biallelic $B A P 1$ missense mutation that would cause functional loss, and also a biallelic SETD2 mutation. In addition, this tumor was negative for BAP1 nuclear staining, although the mutation did not occur in the nuclear-localization signal. Biallelic deletion of $B A P I$ was not detected. The frequency of $B A P 1$ mutation $(11.1 \%)$ is close to that in other studies $(9-11,18)$. Even though the mutation rate of $B A P 1$ was low, $1 / 3$ of our tumors showed negative nuclear staining of BAP1 by immunohistochemistry. Peña-Llopis et al have also reported this difference, but the difference was small because 25 out of 176 samples were negative for nuclear staining, and 22 out of 25 tumors without expression had a BAPl mutation (9). A subsequent multi-institutional cohort study using immunohistochemistry without genetic testing indicated that BAP1 protein was negative for nuclear staining in 82 of 559 ccRCC tumors (14.7\%) (37). Because of the genetic heterogeneity of ccRCC (38), it was difficult to validate heterogeneous staining for BAP1 to obtain uniformity in scoring, without differences among institutions.

There are several ways to explain negative nuclear expression of BAP1 protein without biallelic mutation or deletion: epigenetic regulation of gene expression by DNA methylation or histone modification, excess degradation of mRNA or protein, repressed translation by miRNA, or dysregulation of nuclear localization. Promoter methylation of $B A P l$ was not detected in ccRCC (39) nor in MM (29). Nuclear localization is important for the function of this protein (31). Recently a ubiquitin-conjugating enzyme, UBE2O, has been noted to multi-monoubiquitinate the nuclear localization signal of BAP1, resulting in its cytoplasmic sequestration. UBE2O activity is counteracted by BAP1 autodeubiquitination through intramolecular interactions (32).

Three chRCC tumors showed strong cytosolic staining of BAP1 that might result from copy number amplification of this gene or aberrant transportation by BAP1 ubiquitinated by UBE2O. We could not test the expression level of the $B A P 1$ gene because our RCC samples contained a substantial proportion of non-tumor cells. Because $\sim 30 \%$ of ccRCC tumors with hemizygous normal BAPl showed negative nuclear staining (Fig. 1), haploinsufficiency might be related to RCC development.

BAP1 mutations have been tightly linked to worse clinical outcome in multiple studies $(9,11,40)$. Our data showed that 
patients with biallelic $B A P 1$ mutation had worse recurrencefree survival than the patients without biallelic mutation $(\mathrm{p}=0.046)$, although multivariate analyses did not show the association between $B A P 1$ mutation and tumor recurrence probably due to small sample size. We did not find an association between negative nuclear BAP1 staining and tumor recurrence. This shows that genomic analysis of $B A P 1$ gene is useful in interpreting the prognosis of RCC.

\section{Acknowledgements}

This study was supported in part by a Grant-in-Aid for Researchers from Hyogo College of Medicine, 2013. We are grateful to Atsuko Iemoto and Daiki Haruguchi of Hyogo College of Medicine for their technical assistance.

\section{References}

1. Angeloni D: Molecular analysis of deletions in human chromosome 3p21 and the role of resident cancer genes in disease. Brief Funct Genomics Proteomics 6: 19-39, 2007.

2. Kok K, Naylor SL and Buys CH: Deletions of the short arm of chromosome 3 in solid tumors and the search for suppressor genes. Adv Cancer Res 71: 27-92, 1997.

3. Gnarra JR, Tory K, Weng Y, Schmidt L, Wei MH, Li H, Latif F, Liu S, Chen F, Duh FM, et al: Mutations of the VHL tumour suppressor gene in renal carcinoma. Nat Genet 7: 85-90, 1994.

4. Clifford SC, Prowse AH, Affara NA, Buys CH and Maher ER: Inactivation of the von Hippel-Lindau (VHL) tumour suppressor gene and allelic losses at chromosome arm $3 p$ in primary renal cell carcinoma: Evidence for a VHL-independent pathway in clear cell renal tumourigenesis. Genes Chromosomes Cancer 22: 200-209, 1998.

5. Sükösd F, Kuroda N, Beothe T, Kaur AP and Kovacs G: Deletion of chromosome 3p14.2-p25 involving the VHL and FHIT genes in conventional renal cell carcinoma. Cancer Res 63: 455-457, 2003.

6. Singh RB and Amare Kadam PS: Investigation of tumor suppressor genes apart from VHL on $3 p$ by deletion mapping in sporadic clear cell renal cell carcinoma (cRCC). Urol Oncol 31: $1333-1342,2013$

7. Varela I, Tarpey P, Raine K, Huang D, Ong CK, Stephens P, Davies H, Jones D, Lin ML, Teague J, et al: Exome sequencing identifies frequent mutation of the SWI/SNF complex gene PBRM1 in renal carcinoma. Nature 469: 539-542, 2011.

8. Dalgliesh GL, Furge K, Greenman C, Chen L, Bignell G, Butler A, Davies H, Edkins S, Hardy C, Latimer C, et al: Systematic sequencing of renal carcinoma reveals inactivation of histone modifying genes. Nature 463: 360-363, 2010.

9. Peña-Llopis S, Vega-Rubín-de-Celis S, Liao A, Leng N, PavíaJiménez A, Wang S, Yamasaki T, Zhrebker L, Sivanand S Spence P, et al: BAP1 loss defines a new class of renal cell carcinoma. Nat Genet 44: 751-759, 2012.

10. Guo G, Gui Y, Gao S, Tang A, Hu X, Huang Y, Jia W, Li Z, He M, Sun L, et al: Frequent mutations of genes encoding ubiquitinmediated proteolysis pathway components in clear cell renal cell carcinoma. Nat Genet 44: 17-19, 2011.

11. Sato Y, Yoshizato T, Shiraishi Y, Maekawa S, Okuno Y, Kamura T, Shimamura T, Sato-Otsubo A, Nagae G, Suzuki H, et al: Integrated molecular analysis of clear-cell renal cell carcinoma. Nat Genet 45: 860-867, 2013.

12. Testa JR, Cheung M, Pei J, Below JE, Tan Y, Sementino E, Cox NJ, Dogan AU, Pass HI, Trusa S, et al: Germline BAP1 mutations predispose to malignant mesothelioma. Nat Genet 43: 1022-1025, 2011.

13. Wiesner T, Obenauf AC, Murali R, Fried I, Griewank KG, Ulz P, Windpassinger C, Wackernagel W, Loy S, Wolf I, et al: Germline mutations in BAP1 predispose to melanocytic tumors. Nat Genet 43: 1018-1021, 2011.

14. Abdel-Rahman MH, Pilarski R, Cebulla CM, Massengill JB, Christopher BN, Boru G, Hovland P and Davidorf FH: Germline BAP1 mutation predisposes to uveal melanoma, lung adenocarcinoma, meningioma, and other cancers. J Med Genet 48: 856-859, 2011.
15. Carbone M, Ferris LK, Baumann F, Napolitano A, Lum CA, Flores EG, Gaudino G, Powers A, Bryant-Greenwood P, Krausz T, et al: BAP1 cancer syndrome: Malignant mesothelioma, uveal and cutaneous melanoma, and MBAITs. J Transl Med 10: 179, 2012.

16. Popova T, Hebert L, Jacquemin V, Gad S, Caux-Moncoutier V, Dubois-d'Enghien C, Richaudeau B, Renaudin X, Sellers J, Nicolas A, et al: Germline BAP1 mutations predispose to renal cell carcinomas. Am J Hum Genet 92: 974-980, 2013.

17. Farley MN, Schmidt LS, Mester JL, Peña-Llopis S, PaviaJimenez A, Christie A, Vocke CD, Ricketts CJ, Peterson J, Middelton L, et al: A novel germline mutation in BAP1 predisposes to familial clear-cell renal cell carcinoma. Mol Cancer Res 11: 1061-1071, 2013.

18. Liao L, Testa JR and Yang H: The roles of chromatin-remodelers and epigenetic modifiers in kidney cancer. Cancer Genet 208: 206-214, 2015.

19. Wang SS, Gu YF, Wolff N, Stefanius K, Christie A, Dey A, Hammer RE, Xie XJ, Rakheja D, Pedrosa I, et al: Bap1 is essential for kidney function and cooperates with Vhl in renal tumorigenesis. Proc Natl Acad Sci USA 111: 16538-16543, 2014.

20. Peña-Llopis S, Christie A, Xie XJ and Brugarolas J: Cooperation and antagonism among cancer genes: The renal cancer paradigm. Cancer Res 73: 4173-4179, 2013.

21. Scheuermann JC, de Ayala Alonso AG, Oktaba K, Ly-Hartig N, McGinty RK, Fraterman S, Wilm M, Muir TW and Müller J: Histone $\mathrm{H} 2 \mathrm{~A}$ deubiquitinase activity of the Polycomb repressive complex PR-DUB. Nature 465: 243-247, 2010.

22. Harbour JW, Onken MD, Roberson ED, Duan S, Cao L, Worley LA, Council ML, Matatall KA, Helms C and Bowcock AM: Frequent mutation of BAP1 in metastasizing uveal melanomas. Science 330: 1410-1413, 2010.

23. Ewens KG, Kanetsky PA, Richards-Yutz J, Purrazzella J, Shields CL, Ganguly T and Ganguly A: Chromosome 3 status combined with BAP1 and EIF1AX mutation profiles are associated with metastasis in uveal melanoma. Invest Ophthalmol Vis Sci 55: 5160-5167, 2014.

24. Yoshikawa Y, Sato A, Tsujimura T, Emi M, Morinaga T, Fukuoka K, Yamada S, Murakami A, Kondo N, Matsumoto S, et al: Frequent inactivation of the BAP1 gene in epithelioid-type malignant mesothelioma. Cancer Sci 103: 868-874, 2012.

25. Emi M, Yoshikawa Y, Sato C, Sato A, Sato H, Kato T, Tsujimura T, Hasegawa S, Nakano T and Hashimoto-Tamaoki T: Frequent genomic rearrangements of BRCA1 associated protein-1 (BAP1) gene in Japanese malignant mesothelioma-characterization of deletions at exon level. J Hum Genet 60: 647-649, 2015.

26. Bott M, Brevet M, Taylor BS, Shimizu S, Ito T, Wang L, Creaney J, Lake RA, Zakowski MF, Reva B, et al: The nuclear deubiquitinase BAP1 is commonly inactivated by somatic mutations and 3 p21.1 losses in malignant pleural mesothelioma. Nat Genet 43: 668-672, 2011.

27. Zauderer MG, Bott M, McMillan R, Sima CS, Rusch V, Krug LM and Ladanyi M: Clinical characteristics of patients with malignant pleural mesothelioma harboring somatic BAP1 mutations. J Thorac Oncol 8: 1430-1433, 2013.

28. Arzt L, Quehenberger F, Halbwedl I, Mairinger T and Popper HH: BAP1 protein is a progression factor in malignant pleural mesothelioma. Pathol Oncol Res 20: 145-151, 2014.

29. Nasu M, Emi M, Pastorino S, Tanji M, Powers A, Luk H, Baumann F, Zhang YA, Gazdar A, et al: High incidence of somatic BAP1 alterations in sporadic malignant mesothelioma. J Thorac Oncol 10: 565-576, 2015.

30. Mori T, Sumii M, Fujishima F, Ueno K, Emi M, Nagasaki M, Ishioka $\mathrm{C}$ and Chiba N: Somatic alteration and depleted nuclear expression of BAP1 in human esophageal squamous cell carcinoma. Cancer Sci 106: 1118-1129, 2015.

31. Yu H, Mashtalir N, Daou S, Hammond-Martel I, Ross J, Sui G, Hart GW, Rauscher FJ III, Drobetsky E, Milot E, et al: The ubiquitin carboxyl hydrolase BAP1 forms a ternary complex with YY1 and HCF-1 and is a critical regulator of gene expression. Mol Cell Biol 30: 5071-5085, 2010.

32. Mashtalir N, Daou S, Barbour H, Sen NN, Gagnon J, HammondMartel I, Dar HH, Therrien M and Affar B: Autodeubiquitination protects the tumor suppressor BAP1 from cytoplasmic sequestration mediated by the atypical ubiquitin ligase UBE2O. Mol Cell 54: 392-406, 2014.

33. Dietrich W, Katz H, Lincoln SE, Shin HS, Friedman J, Dracopoli NC and Lander ES: A genetic map of the mouse suitable for typing intraspecific crosses. Genetics 131: 423-447, 1992. 
34. Cingolani P, Platts A, Wang L, Coon M, Nguyen T, Wang L, Land SJ, Lu X and Ruden DM: A program for annotating and predicting the effects of single nucleotide polymorphisms, SnpEff: SNPs in the genome of Drosophila melanogaster strain w1118; iso-2; iso-3. Fly (Austin) 6: 80-92, 2012.

35. Liu X, Jian X and Boerwinkle E: dbNSFP v2.0: A database of human non-synonymous SNVs and their functional predictions and annotations. Hum Mutat 34: E2393-E2402, 2013.

36. Herman JG, Graff JR, Myöhänen S, Nelkin BD and Baylin SB: Methylation-specific PCR: A novel PCR assay for methylation status of CpG islands. Proc Natl Acad Sci USA 93: 9821-9826, 1996.

37. Kapur P, Christie A, Raman JD, Then MT, Nuhn P, Buchner A, Bastian P, Seitz C, Shariat SF, Bensalah K, et al: BAP1 immunohistochemistry predicts outcomes in a multi-institutional cohort with clear cell renal cell carcinoma. J Urol 191: 603-610, 2014.
38. Gerlinger M, Horswell S, Larkin J, Rowan AJ, Salm MP, Varela I, Fisher R, McGranahan N, Matthews N, Santos CR, et al: Genomic architecture and evolution of clear cell renal cell carcinomas defined by multiregion sequencing. Nat Genet 46: 225-233, 2014

39. Ibragimova I, Maradeo ME, Dulaimi E and Cairns P: Aberrant promoter hypermethylation of PBRM1, BAP1, SETD2, KDM6A and other chromatin-modifying genes is absent or rare in clear cell RCC. Epigenetics 8: 486-493, 2013.

40. Hakimi AA, Ostrovnaya I, Reva B, Schultz N, Chen YB, Gonen M, Liu H, Takeda S, Voss MH, Tickoo SK, et al; ccRCC Cancer Genome Atlas (KIRC TCGA) Research Network investigators: Adverse outcomes in clear cell renal cell carcinoma with mutations of 3p21 epigenetic regulators BAP1 and SETD2: A report by MSKCC and the KIRC TCGA research network. Clin Cancer Res 19: 3259-3267, 2013. 